\title{
Container truck transportation routing as a Mixed Fleet Heterogeneous Dial-a-Ride Problem
}

\author{
Mohamed A mine M asmoudi ${ }^{1}$, Katarzyna Anna $\mathrm{K}$ uzmicz $^{2 *}$, Erwin Pesch $^{3}$, Emrah Demir ${ }^{4}$ \\ and Manar Hosny ${ }^{5}$ \\ ${ }^{1}$ Geneva School of Business A dministration, U niversity of A pplied Sciences W estern Switzerland \\ (HES-SO) 1227 Carouge, Switzerland \\ ${ }^{2}$ International China and Central-E astern Europe Institute of L ogistics and Service Science, Faculty \\ of Engineering M anagement, Bialystok U niversity of Technology, ul. W iejska 45 A, 15-351 \\ Bialystok, Poland \\ ${ }^{3}$ Department of Management Information Science, University of Siegen, Hölderlinstraße 3, D-57068 \\ Siegen, Germany, E-mail: erwin.pesch@ uni-siegen.de and Centre for A dvanced Studies in \\ M anagement, HHL L eipzig Graduate School of M anagement, J ahnallee 59, 04109 L eipzig, Germany \\ ${ }^{4}$ Panal pina Centre for M anufacturing and L ogistics Research, Cardiff B usiness School, Cardiff \\ University, Cardiff CF10 3EU, UK \\ ${ }^{5}$ Computer Science Department, College of Computer and Information Sciences (CCIS), K ing Saud \\ University (KSU), Riyadh, Saudi A rabi
}

\begin{abstract}
In this paper, a new variant of the Heterogeneous Dial-a-Ride Problem (HDARP) is innovatively applied to a routing problem of trucks picking up and delivering full and empty containers in the logistic chain, including container depots, importers demanding full containers and exporters requesting empty containers. $0 \mathrm{n}$ the route different types of trucks pick-up and deliver full and empty 20 - and 40 -foot containers. We consider trucks that are operated by alternative fuel, as well as the need for refuelling of trucks in any alternative fuel station to eliminate the risk of running out of fuel during its route. The objective of the paper is to contribute to the optimization of container truck transport routing with multiple pickups and deliveries by providing a mathematical programming model aiming at minimizing the transportation cost as well as the negative environmental impacts.
\end{abstract}

Keywords: dial-a-ride problem, full and empty containers, green vehicle routing, intermodal transportation

* Corresponding author: k.kuzmicz@pb.edu.pl 


\section{Introduction}

Container transportation on long distances is performed by sea and rail. On short distances between ports, transhipment terminals, container depots, exporters' and importers' containers are transported by trucks. The Organisation for Economic Cooperation and Development OECD (54 member countries) predicted global trade-related freight and emissions to quadruple by 2050 . As the majority of goods are transported in containers, the container traffic on the road will rise significantly. Therefore a problem of optimal routing of trucks, transporting containers, especially with consideration of environmental issues is worth attention.

In container truck transportation the following three basic types of service can be differentiated $[1,2]$ :

- an order request from an importer for moving a full container from an origin terminal to a given location, where the cargo is unloaded from the container, and then to move the empty container to its destination;

- an order request from an exporter to move an empty container from an origin terminal or a depot to a given location, where some freight is loaded into the container, and then to move the full container to its destination, mostly a terminal;

- a request of transporting an empty container from a given container terminal to an empty container depot, or vice versa.

For each order, hard time windows may be present at each of the visited locations.

After a pickup request for an emptied container has been conducted, an empty container is released at the destination location (where the cargo was unloaded) and is either transported to a depot or to the origin location of a delivery request for an empty container. Thus, each pickup request has to be followed by a delivery request/depot. Similarly, each delivery request requires an empty container at the origin location and either receives an empty container from a depot or a pickup request, i.e., a pickup request/depot has to precede a delivery request. This empty container assignment problem is solved in the first phase of the method by Nossack and Pesch[1,2] by constructing pickup and delivery pairings such that each pickup and delivery request is paired exactly once and each pairing is feasible related to the time windows.

An excellent survey on the fundamental problem of empty container repositioning in intermodal transport supply chains, embracing trade route between Asia and China in particular, can be found in Kuzmicz and Pesch [3].

Trucks can transport one or two containers. The maximum weight of a loaded module (truck-trailer) is restricted by national law depending on the type of road used for transportation. It is estimated that transportation of two heavy containers, despite the heavy pressure on the road, is more environmentally friendly than transporting each container separately, and can contribute to $\mathrm{CO}_{2}$ emission reduction even by $35 \%$ considering the amount of $\mathrm{CO}_{2}$ to the amount of load transported [4]. Consequently, it also contributes to lower fuel consumption.

In the DARP it is assumed that Conventional Vehicles (CVs) are used to serve the customers. In classical routing problems, it is usually assumed that Conventional V ehicles (CV s) are used to serve the requests. Furthermore, it is normally assumed that CV $\mathrm{s}$ have unlimited fuel tank capacity; thus, there is no need for the vehicle to refuel during its service route to customers. H owever, CV s are one of the main contributors to emissions, such as greenhouse gases (GHGs) and air pollutants. In fact, the European Environment A gency (EEA) has estimated that transportation is responsible for approximately $22 \%$ of GHG emissions in 2012. To overcome the environmental challenges as well as the limited energy resource concerns, the transport industry looks for solutions that make use of $A$ Iternative Fuel $\checkmark$ ehicles (AFV s) (i.e., al ternative fuel trucks), such as biodiesel or ethanol operated vehicles. 
It is obvious that AFV s can help in reducing GHGs emissions compared to CV s that use gasoline or diesel [5]. In this regard, A FV s have started to gain attention in the last few years, due to their benefits in the field of transportation routing (see, e.g., $[5,6,7]$. Thus, this has inspired us to consider the use of A FV s in the current work within the context of the container routing problem.

The routing of container transportation service is exemplified in the Figure1.

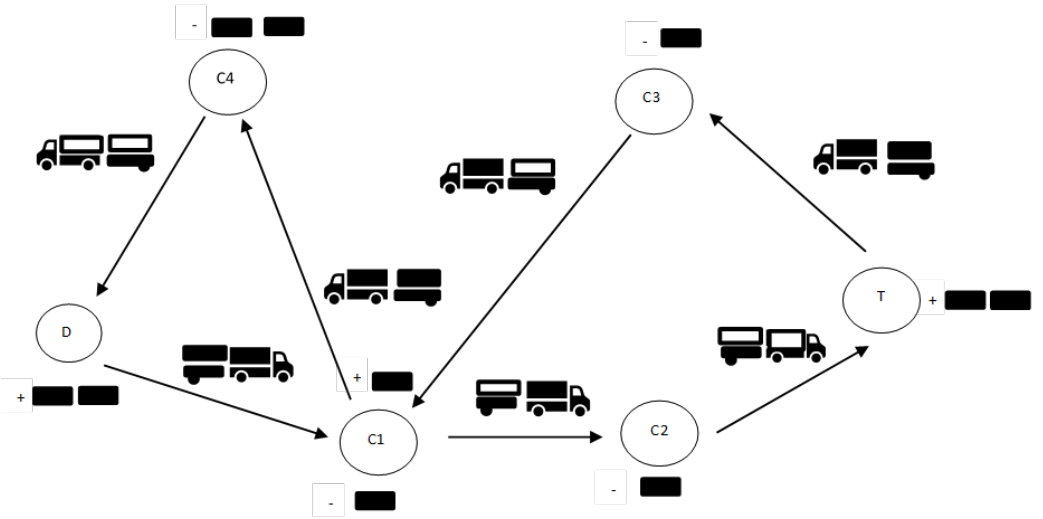

Figure 1. Container truck transportation routing

In the exemplified case a truck picks up two loaded containers at a terminal at a depot or a terminal D and visits client $\mathrm{C} 1$ where one container is emptied. Later it continues with one full and one empty container. At client $\mathrm{C} 2$ the remaining full container is emptied and the truck continues with two empty containers to container terminal T. At container terminal $\mathrm{T}$ two empty containers are left and two full containers are picked up. The next stop is at client $\mathrm{C} 3$ where one container is emptied. Later the truck visits once more client $\mathrm{C} 1$ where the empty container is filled. With two full containers, it goes to client $\mathrm{C} 4$ where the two full containers are emptied. The truck finishes its route at depot D with two empty containers. Note that a ' + ' and a '-' next to a node of the network indicates whether loaded containers are picked up or dropped off, respectively.

Different models can be used for container truck routing. Here a Dial-a-Ride Problem (DARP) has been applied. Typically applications serve to optimize transportation of elder and/or disabled people with reduced mobility but also in shared taxi services [8] or courier services [9] with multiple pick-up and delivery.

The contribution of this paper includes mathematical modelling of the aforementioned truck routing problem as a heterogeneous dial-a-ride problem with a mixed fleet and refuelling of vehicles.

The remainder of the paper is the following. First, the general idea and standard application of a DARP is presented, later a problem description of HDARP applied to routing of a container pick-up and delivery in the logistic chain of container depots, terminals, exporters and importers are described. Then a mathematical model is proposed followed by a short description of computational experiments.

\section{The heterogenous dial-a ride-problem}

The DARP involves designing vehicle routes and schedules for different users who specify pick-up and delivery requests between origins and destinations under a number of constraints, typically concerning trip duration, time windows, and vehicle capacity. It is a generalisation of a number of vehicle routing problems such as the Pick-up and Delivery 
Vehicle Routing Problem (PDVRP) and the Vehicle Routing Problem with Time Windows (VRPTW) [10]. The specificity of this problem relies on variable vehicle capacity. There is a static and dynamic version of DARP based on schedules fully planned in advance, and with consideration of requests incoming on the day of the service. However, in reality, it is difficult to imagine a pure static or pure dynamic situation because most of the requests would be scheduled in advance but some requests for moving a container could be placed on the day of the service.

DARP with Heterogeneous users and/or alternative fuel vehicles called HDARP [11] is a generalization of the DARP, but it has not been extensively studied in the literature. The first time a formal definition of the HDARP was introduced by [11]. The authors consider two types of conventional fuel vehicles and four different resources (i.e., staff seat, patient seat, stretcher, and wheelchair). They propose a Branch-and-Cut (B\&C) and Variable Neighborhood Search (VNS) algorithms and solved 36 instances with up to four vehicles and 48 requests. In a related study [12] introduce a variant of the HDARP, in which the requirements like lunch break constraints are considered. In Braekers et al. [13], multiple depots of heterogeneous vehicles and users are considered to reduce the total routing costs. The authors propose a B\&C and a Deterministic Annealing metaheuristic to solve instances containing 2-8 vehicles and 16-96 requests. More recently, Masmoudi et al. [14] apply a hybrid Genetic Algorithm (GA) to the standard HDARP. The proposed algorithm was tested on small and medium benchmark instances for the HDARP with up to 8 vehicles and 96 requests proposed by Parragh [11] and Braekers et al. [13]. To best of our knowledge, the hybrid GA provides the best-known results on these instances so far and outperforms current state-of-the-art algorithms for the standard DARP and HDARP. In another study, Masmoudi et al. [15] augment the multi depots and coffee break concepts on the standard HDARP. They propose hybrid methods based on nature-inspired algorithms and tested them on both newly generated instances and on the benchmark Multi-Depot HDARP instances of Braekers et al. [13]. Recently, Braekers and Kovacs [16], extend the single period HDARP to a Multi-Period DARP and consider a limited number of drivers to serve each user over a predefined number of days.

For other variants of (H)DARP applied to real concepts the interested readers are referred to surveys on the DARP by Cordeau and Laporte [10] and Molenbruch et al. [17].

Container transportation is performed by a mixed fleet of different types of vehicles. We assume usage of trucks carrying 20-foot containers and tractors with semi-trailers carrying one 40 -foot container or two 20 -foot containers or one 20 -foot and one 40 -foot container (see Figure 2).

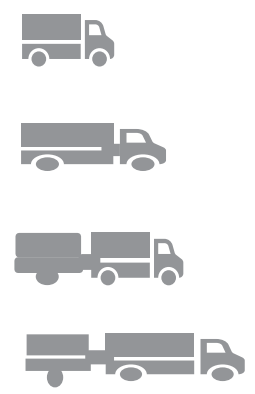

\author{
a truck with a 20 -foot \\ a tractor with a semi-trailer carrying \\ a 40-foot container \\ a tractor with a semi-trailer carrying \\ two 20-foot containers \\ a tractor with a semi-trailer \\ carrvina a 40-foot container
}

Figure 2. Trucks and tractors with semi-trailers carrying containers

Fuel consumption is dependent on this construction of the transportation module and on the weight of the containers and whether they are transported full or empty. 
Consolidating containers and limiting the number of trucks leads generally to lowering the fuel consumption needed for the transportation task.

Due to the limited driving ranges of the AFVs (around 120 miles) because of their limited tank capacity [5], these types of vehicles need to be refuelled from Alternative Fueling Stations (AFSs). In fact, in the context of the DARP, not taking the refuelling requirements beforehand in planning the service route may cause service disruption due to a shortage in fuel, and possible violation of the problem constraints, such as the visiting time windows and/or the maximum ride time of drivers. Such violations can largely lead to the dissatisfaction of customers, which impacts the overall service quality and breaks one of the main conditions of the DARP. Therefore, effective transportation planning should take into consideration the visits of users as well as visits to AFSs, since the need to refuel the tank is frequently encountered during the customary working day, especially when travelling long distances [5].

Another realistic assumption in routing problems is the use of an appropriate fuel consumption function based on several factors, such as vehicle speed and load ( e.g., [18, 19, $20,21])$.

\section{The green vehicle routing problem}

Sanguinetti et al. [22] indicate six classes of eco-driving actions, driving, cabin comfort, trip planning, load management, fuelling, and maintenance.

The Green Vehicle Routing Problem (GVRP) with consideration of the alternative fuel tank capacity limitation is an extension of the VRP that has received increasing attention recently. Erdoğan and Miller-Hooks [5] were the first authors that introduced the GVRP, where refuelling stops are incorporated. The authors proposed a mixed-integer linear model to minimize the travel distance and developed two efficient heuristics and tested them on instances with up to 500 users. Other studies on GVRP with refuelling can be found on Felipe et al. [23] and Adler and Mirchandani [6]. An in-depth investigation of GVRP is developed by Lin et al. [24].

The incorporation of real fuel consumption and emission in the context of the green vehicle routing (GVR) has been widely studied in the recent literature, where these factors play an important role in routing models, taking into account fuel costs and vehicles' emissions (see, e.g., Bektaş and Laporte [18]; Demir et al. [19] and Goeke and Schneider, [25]; Franceschetti et al. [26]; and Androutsopoulos and Zografos [27]. Demir et al. [21], [28]) performed a thorough study of the energy consumption of related routing problems.

Although the concept of heterogeneous vehicles and/or users have been already studied within the context of $(\mathrm{H}) \mathrm{DARP}$, what distinguishes our study is that we consider a mixed fleet of trucks that differs in their capacity.

\section{Problem definition and model formulation}

The Container Mixed Fleet Heterogeneous Dial-a-Ride Problem (CMF-HDARP) can be formally described as follows. Consider a graph $G=(V, A)$ with node set $V$ and arc set $=\{(i, j): i, j \in V, i \neq j$, where $V$ is further partitioned into subsets; $N=\{1, \ldots, 2 n\}$ corresponds to $n$ users requesting a transport service. In other words, a user can be identified with an ordered pair of nodes $(i, j), i \neq j$, where $i$ is the pick-up location of a loaded container and $j$ or, for convenience $n+i$, is the predefined drop-off/delivery location. Alternatively, we say container $i$ is picked up at location $i$ and delivered to its destination $n+i$. If the container is empty, one of the two locations might be a result of the optimization. 
Let $P=\{1, \ldots, n\}$ and $D=\{n+1, \ldots, 2 n\}$ be the not necessarily disjoint subsets of nodes corresponding to pick-up and delivery locations, respectively. Moreover, $F=\{2 n+$ $1, \ldots, 2 n+f\}$ defines the set of Alternative Fuel Stations (AFSs). Nodes 0 and $2 n+1+$ $f$ correspond to the origin and destination depots, respectively. Thus, we get $V=N \cup F \cup$ $\{0,2 n+1+f\}$. There is a non-negative travel cost $c_{i j}$, depending on travel speed $v_{i j}$ and transportation time $t_{i j}$ associated with each arc $(i, j)$ from set $A$. We assume the speed to be constant over an arc $(i, j)$ as done in Goeke and Schneider [25], and the number of stops that can be made for refuelling is unlimited. When refuelling takes place, it is assumed that the tank is refilled to its maximum capacity. The time windows to visiting any refuelling node are set as $[0, T]$, where $T$ is the length of the planning horizon. Moreover, a mixed fleet of heterogeneous vehicles $K=\left\{k_{1}, \ldots, k_{k}\right\}$ composed of trucks and tractors with semitrailers is available to transport all containers.

We note that in the mathematical model there is a difference between visits to users' nodes and visits to AFSs nodes/depot, where user nodes must be visited exactly once, while AFS nodes may be visited more than once or not visited at all. Also, the depot must be visited at the start and end of the tour, and can also be considered as an AFS node. Moreover, in order to allow some vertices to be visited more than once, while others are visited exactly once, we augment a set of dummy vertices $f^{\prime}$ on the graph $G$, such that $F^{\prime}=\{2 n+f+1, \ldots, 2 n+f+$ $\left.f^{\prime}\right\}$ (see, e.g., Erdoğan and Miller-Hooks [5]; Goeke and Schneider [25]. Each of these nodes represents a potential visit to an AFS or depot. Thus, the graph $G^{\prime}=\left(V^{\prime}, A^{\prime}\right)$ will consist of $V^{\prime}=V \cup F^{\prime}$.

Each truck has a capacity $Q^{r, k}$ that gives the amount of resource $r$ available on each vehicle $k$, where each type of resource is dedicated to: the full 20 -foot container $Q^{0, k}$, full 40-foot container $Q^{1, k}$, empty 20-foot container $Q^{2, k}$ and empty 40-foot container $Q^{3, k}$. Each vehicle type contains a fuel tank capacity $H^{k}$, which is consumed and reduced at a fuel rate $F R_{i j}$ on each travelled arc $(i, j)$. Each pick-up location $i$ is associated with a demand requirement of transport space $-2 \leq q_{i}^{r} \leq 2$ for each resource $r$, and a time window $\left[T_{i}^{-}, T_{i}^{+}\right]$, where $T_{i}^{-}$and $T_{i}^{+}$represent the earliest and latest visiting time, respectively, of this location. A positive value $q_{i}^{r}$ indicates that there is a request for an empty truck position suitable to capture a container at location $i$. A negative demand value means, a container is dropped from the truck and the position becomes available for a pickup of another container either at the same location or at a subsequent one on the route. A value of zero either indicates that there is no container demand or supply at location $i$ or the number of containers of the same type (resource) dropped and picked at this location is the same. A maximum container transportation time $L_{\max }$ is considered as a service promise for maximum waiting time. In addition, a service time $s_{i}$ is imposed when visiting each node $(\forall i \in N)$, and a refuelling time $s_{f}$ is considered when visiting a recharging station node $\left(\forall f \in F^{\prime}\right)$.

As studied in Demir et al. [19], for the fuel consumption rate of the vehicles, the constant fuel rate $F R_{i j}$ required on each arc $(i, j)$ can be calculated as a function of the mechanical power and a couple of other parameters.

In summary, the CMF-HDARP consists of determining a set of routes to satisfy the users' demands while minimizing the total routing costs. A solution must have the following conditions: $i$ ) the pick-up node must be visited before the corresponding delivery node, $i$ ) the total demand for the route must not exceed the capacity of each resource of the vehicle, iii) each node must be served within its time window; if a vehicle arrives early, it must wait until the beginning of the service, $i v$ ) the maximum transportation time of each container must be respected, $v$ ) the pick-up and delivery nodes of each container must be visited by the 
same vehicle, vi) each vehicle must be refuelled at any AFS node if the remaining tank fuel level is not enough to visit any node $i \in V$, and vii) each route should start and end at the same depot and the route has a maximum duration of $T_{\text {max }}$.

Now we present the integer programming formulation for the CMF-HDARP, which is based on Cordeau [29] and inspired by the formulations of the HDARP of Parragh [11] and the GVRP with the refuelling of Erdoğan and Miller-Hook [5]. The CMF-HDARP can be formulated as follows: Binary variable $x_{i j}^{k}$ is equal to 1 if vehicle $k$ travels from node $i$ to $j$ and 0 otherwise. Variable $Q_{i}^{r k}$ indicates the load of resource $r$ on vehicle $k$ immediately after visiting node $i$. Continuous variable $B_{i}^{k}$ represents the time that the vehicle $k$ begins its service at node $i$. Moreover, continuous variable $l_{i}^{k}$ represents the transportation time of loaded container (from location) $i \in P$ on vehicle $k$. Continuous variable $z_{i}^{k}$ represents the remaining fuel level in the tank upon arrival to node $i$ with vehicle $k$. It is noted that $z_{i}^{k}$ is set to $H^{k}$ at each visit to any alternative fuel station node $f \in F^{\prime}$.

M inimize $\sum_{k \in K} \sum_{i \in V} \sum_{c_{j} \in V} c_{i j} x_{i j}^{k}$

Subject to

$\sum_{k \in K} \sum_{j \in N} x_{i j}^{k}=1$

$\forall i \in P$

$\sum_{k \in K} \sum_{j \in N} x_{j i}^{k}=1$

$\forall i \in D$

$\sum_{j \in N} x_{i j}^{k}=\sum_{j \in N} x_{j, n+i}^{k}$

$\forall k \in K, \forall i \in P$

$\sum_{j \in V \Theta} x_{0 j}^{k}=1$

$\forall k \in K$

$\sum_{i \in V \circlearrowright} x_{i, 2 n+1+f}^{k}=1$

$\forall k \in K$

$\sum_{j \in V \circlearrowleft} x_{j i}^{k}=\sum_{j \in V} x_{\overparen{C}}^{k}$

$\forall k \in K, \forall i \in V \odot$

$Q_{j}^{r k} \geq\left(q_{j}^{r}+Q_{i}^{r k}\right) x_{i j}^{k}$

$\forall k \in K, \forall(i, j) \in A, r \in\{0,1,2,3\}$

$0 \leq Q_{i}^{r k} \leq Q^{r k}$

$\forall k \in K, \forall i \in N, r \in\{0,1,2,3\}$

$Q_{0}^{r k}=0$

$\forall k \in K, r \in\{0,1,2,3\}$

$l_{i}^{k}=B_{n+i}^{k}-\left(B_{i}^{k}+s_{i}\right)$

$\forall k \in K, \forall i \in P$

$t_{i, n+i} \leq l_{i}^{k} \leq L_{\max }$

$\forall k \in K, \forall i \in P$

$B_{j}^{k} \geq\left(B_{i}^{k}+s_{i}+t_{i j}\right) x_{i j}^{k}$

$\forall k \in K, \forall(i, j) \in A$ ๑

$T_{i}^{-} \leq B_{i}^{k} \leq T_{i}^{+}$

$\forall k \in K, \forall i \in V \odot$

$B_{2 n+1+f}^{k} \mathrm{O}^{k} B_{0}^{k} \leq T_{\max }$

$\forall k \in K, \forall f \in F \odot$

$z_{j}^{k}-z_{i}^{k}+M_{i j} x_{i j}^{k}+\left(M_{i j}-F R_{i j} c_{i j}-F R_{i j} c_{j i}\right) x_{j i}^{k} \leq M_{i j}-F R_{i j} c_{i j}$

$\forall i, j \in N, i \neq j, \forall k \in K$

$z_{j}^{k}=H^{k}$

$\forall j \in F @ \forall k \in K$

$z_{j}^{k} \geq \min \left\{F R_{i j} c_{j 0}, F R_{i j}\left(c_{i f \odot}+c_{f 0}\right)\right\}$

$\forall j \in V, \forall f \in F @ \forall k \in K$

$x_{i j}^{k} \in\{0,1\}$

$\forall k \in K, \forall(i, j) \in A \odot$

The objective function (1) minimizes the total routing costs assuming that the travel costs are proportional to the travelled distance. Constraints (2)-(4) guarantee that each pick-up and delivery location is served by a vehicle. Constraints (5)-(6) ensure that each vehicle $k$ starts at the origin depot and ends at the corresponding destination depot, while constraints (7) satisfy flow conservation. Constraints (8) and (9) enforce the consistency with respect to vehicle capacity constraints. Constraints (10) ensure that the load variable on the depot is set to zero, which means that the vehicles leave the depot with empty load. This can 
be adjusted to values one or two if empty containers are picked there. Constraints (11) define the ride time of each container transported from $i$ to $n+1$ in each route, which is bounded by constraints (12). These constraints also impose the precedence relation between the pickup and delivery nodes of a user. Constraints (13) define the beginning of service at each node and the consistency of the time variables. Constraints (14) impose time windows compliance. Constraints (15) ensure that the time duration of the route for each vehicle is limited by $T_{\max }$. Constraints (16) impose that the fuel level is reduced based on the distance travelled between nodes $i$ and $j$ and the fuel consumption rate, where $M_{i j}$ is a constant applied to each $\operatorname{arc}(i, j)$ $\forall i, j \in A^{\prime}$. Constraints (17) guarantee that the fuel level is reset to its maximum $H^{k}$ after visiting each recharging station node. Constraints (18) guarantee that the remaining tank fuel level of the vehicle is enough to return to the initial depot or to reach a fuel station from any user location. Finally, constraints (19) guarantee that the decision variables are binary.

\section{Conclusions}

The problem is certainly NP-hard as dial-a-ride problems are NP-hard. However, it is a challenge to see how far a standard solver can get in solving instances of these problems. Initial implementation in CPLEX is intended which then will be enriched by a decomposition. Earlier experiments let us hope that competitive results for smaller to medium-sized problems can be achieved within an hour of run time.

Container truck transportation routing with several pick-up and delivery with optimizing transportation cost, fuel consumption and pollution emission is a topical problem requiring further investigation. In this paper, a new and more realistic version is proposed and its application to container truck transportation can be perceived as innovative. We considered a mixed fleet of vehicles (Alternative Fuel Vehicles) composed of both alternative fuel trucks and tractors with semi-trailers subject to constraints of capacity of transporting 20-foot and 40-foot full or empty containers including refuelling of vehicles.

Our model is limited in that respect that the vehicles will be refuelled in each visit to the station.

In a centralized decision process, as mentioned above, a global optimum maximizes a kind of social welfare while different transport requests do not equally contribute to the emissions and pollution. As a consequence, the clients wish to participate in the development of a fair solution and fair pricing strategy. Therefore the transport company might develop a mechanism (a game) so that all clients can reach a satisfying solution, see Kress et al. [30, 31]. In the ideal case, the mechanism encourages the clients (or players) to provide truthful information in any negotiation process with the transport company or competitor and to discourage selfish behaviour. A mechanism is needed where selfish behaviour does not provide any advantage in comparison to truth-telling. Moreover, the clients have insight and understand the functions of the mechanism. In equilibrium, no client would like to change its position in the transport sequence and the transport company would focus on those equilibria that maximize social welfare. For details, see Kovalyov and Pesch [32]. Fast and efficient methods need to be developed in order to find an equilibrium as this might be a strongly NP-hard problem or good approximations of equilibrium are to be found.

\section{Acknowledgements}

This study was supported by Bialystok U niversity of Technology (S/W Z/1/2014). 


\section{References}

1. J. N ossack, E. Pesch, A truck scheduling problem arising in intermodal container transportation, European Journal of Operational Research 230: 666-680 (2013a)

2. J. Nossack, E. Pesch, Planning and scheduling in intermodal transport, Manufacturing Modelling, Management and Control 2013 in The Proceedings of the IFA C International Conference, St Petersburg, Russia (2013) (2013b)

3. K.A. K uzmicz, E. Pesch, A pproaches to empty container repositioning problems in the context of Eurasian intermodal transportation, Omega - The Int. Journal of Management Science, in press. (2018)

4. 40ton.net, https://40ton.net/dwa-ciezkie-kontenery-20-stopowe-w-ramach-az-74tonowego-zestawu-jak-najbardziej-moga-miec-sens

5. S. Erdoğan, E. M iller-Hooks, A green vehicle routing problem, Transportation Research Part E: Logistics and Transportation Review 48: 100-114 (2012)

6. J.D. A dler, P.B. M irchandani, The vehicle scheduling problem for fleets with alternative-fuel vehicles, Transportation Science 51: 441-456 (2016)

7. Ç. Koç, I. Karaoglan, The green vehicle routing problem: A heuristic based exact solution approach, Applied Soft Computing 39, 154-164 (2016)

8. D.O. Santos, E.C. Xavier, Taxi and Ride Sharing: A Dynamic Dial-a-Ride Problem with M oney as an Incentive, Expert Systems with Applications 42: 6728-6737 (2015) 8

9. Hunsaker, M.V.P. Savelsbergh, Efficient feasibility testing for dial-a-ride problems, Operations Research Letters 30: 169-173 (2012)

10. J.-F. Cordeau, G. Laporte. The dial-a ride problem: models and algorithms, Annals of Operations Research 153: 29-46 (2007)

11. S.N. Parragh, Introducing heterogeneous users and vehicles into models and algorithms for the dial-a-ride problem. Transportation Research Part C: Emerging Technologies 19: $912-930$ (2011)

12. S.N. Parragh, J.F. Cordeau, K. F., Doerner, R. F., Hartl, M odels and algorithms for the heterogeneous dial-a-ride problem with driver-related constraints, OR Spectrum 34, 593-633 (2012)

13. K. B raekers. A. Caris, G.K. J anssens, Exact and meta-heuristic approach for a general heterogeneous dial-a-ride problem with multiple depots, Transportation Research Part B: Methodological 67: 166-186 (2014)

14. M.A. M asmoudi, K. B raekers, M. M asmoudi, A. Dammak, A hybrid genetic algorithm for the heterogeneous dial-a-ride problem, Computers \& Operations Research 81: 1-13 (2017)

15. M.A. M asmoudi, M. Hosny, K. Braekers, A. Dammak, Three effective metaheuristics to solve the multi-depot multi-trip heterogeneous dial-a-ride problem, Transportation Research Part E: Logistics and Transportation Review 96: 60-80 (2016)

16. K. B raekers, A. K ovacs, A multi-period dial-a-ride problem with driver consistency. A multi-period dial-a-ride problem with driver consistency, Transportation Research Part B: Methodological 94: 355-377 (2016)

17. Y. M olenbruch, K. B raekers, A. Caris, Typology and literature review for dial-a-ride problems, Annals of Operations Research 259: 295-325 (2017)

18. T. B ektas, G. L aporte, The pollution-routing problem. Transportation Research Part B: Methodological 45: 1232-1250 (2011) 
19. E. Demir, T. B ektas, G. Laporte, An adaptive large neighborhood search heuristic for the pollution-routing problem, European Journal of Operational Research 223: 346359 (2012)

20. E. Demir, T. B ektas, G. L aporte, The bi-objective pollution-routing problem. European Journal of Operational Research, 232: 464-478 (2014a)

21. E. Demir, T. B ektas, G. Laporte, A review of recent research on green road freight transportation. European Journal of Operational Research 237: 775-793 (2014b)

22. A. Sanguinetti, K. K urani, J. Davies, The many reasons your mileage may vary: Toward a unifying typology of eco-driving behaviours, Transportation Research Part D: Transport and Environment 52 (Part A), 73-84 (2017)

23. Á. Felipe, M.T. Ortuño, G. Righini, G. Tirado, A heuristic approach for the green vehicle routing problem with multiple technologies and partial recharges. Transportation Research Part E: Logistics and Transportation Review 71: 111-128 (2014)

24. C. Lin, K.L. Choy, G.T.S. Ho, S.H. Chung, H.Y. Lam, Survey of Green Vehicle Routing Problem: Past and future trends, Expert Systems with Applications 41: 11181138 (2014)

25. D. Goeke, M. Schneider, R outing a mixed fleet of electric and conventional vehicles, European Journal of Operational Research 245, 81-99 (2015)

26. A. Franceschetti, E. Demir, D. Honhon, T. V an W oensel, G. Laporte, M. Stobbe, A metaheuristic for the time-dependent pollution-routing problem, European Journal of Operational Research 259(3): 972-991 (2017)

27. K.N. A ndroutsopoulos, K.G. Zografos, A n integrated modelling approach for the bicriterion vehicle routing and scheduling problem with environmental considerations, Transportation Research Part C: Emerging Technologies 82:180-209 (2017)

28. E. Demir, Y. Huang, S. Scholts, T. V an W oensel, A selected review on the negative externalities of the freight transportation: M odeling and pricing, Transportation Research Part E: Logistics and Transportation Review 77: 95-114 (2015)

29. J.-F. Cordeau, A branch-and-cut al gorithm for the dial-a-ride problem. Operations Research 54: 573-586 (2016)

30. D. K ress, S. M eiswinkel, E. Pesch, M echanism design for machine scheduling problems, OR Spectrum 40: 583-611 (2018a)

31. D. K ress, S. M eiswinkel E. Pesch, Incentive compatible mechanisms for machine scheduling problems with job agents, Discrete Applied Mathematics 242: 89-101 (2018b)

32. M.Y. K ovalyov, E. Pesch, A game mechanism for single machine sequencing with zero risk, Omega - The Int. Journal of Management Science 44, 104-110 (2014) 